\title{
Selective suppression of autocatalytic caspase-3 driven by two-step transcriptional amplified human telomerase reverse transcriptase promoter on ovarian carcinoma growth in vitro and in mice
}

\author{
YUE SONG $^{1}$, XING XIN $^{1}$, ZHIJUN XIA $^{1}$, XINGYUE ZHAI $^{1}$ and KENG SHEN ${ }^{2}$ \\ ${ }^{1}$ Department of Obstetrics and Gynecology, Shengjing Hospital, China Medical University, Shenyang 110004; \\ ${ }^{2}$ Department of Obstetrics and Gynecology, Peking Union Medical College Hospital, Peking Union \\ Medical College, Chinese Academy of Medical Sciences, Beijing 100730, P.R. China
}

Received March 17, 2014; Accepted May 2, 2014

DOI: $10.3892 / o r .2014 .3204$

\begin{abstract}
The objective of our study was to construct recombinant adenovirus (rAd) AdHTVP2G5-rev-casp3, which expresses autocatalytic caspase-3 driven by human telomerase reverse transcriptase promoter (hTERTp) with a two-step transcription amplification (TSTA) system and investigate its antitumor effects on ovarian cancer in vitro and in vivo. Fluorescent detection was used to detect EGFP expression in various cells. Cell viabilities were determined using the Cell Counting Kit- 8 and flow cytometry. RT-PCR and immunoblotting assays were used to detect cellular apoptotic activities. Tumor growth and survival of tumor-bearing mice were studied. The hTERTp-TSTA system showed the strongest activity in hTERT-positive cancer cells when compared with hTERTp and cytomeglovirus promoter (CMVp). In contrast, it showed no activity in hTERT-negative HUVECs. AdHTVP2G5-rev-casp3 markedly suppressed the survival of $\mathrm{AO}$ cells in a dose-dependent modality with a viability rate of $17.8 \pm 3.5 \%$ at an MOI of 70 , which was significantly lower than that by AdHT-rev-casp3 and Ad-rev-casp3 (rAds which express rev-caspase- 3 driven by hTERTp and CMVp, respectively). In contrast, AdHTVP2G5-rev-casp3 induced little HUVEC death with a viability rate of $92.7 \pm 5.2 \%$ at the same MOI. Additionally, AdHTVP2G5-rev-casp3 (MOI=70) caused significant apoptosis in AO cells with an apoptotic rate of $42 \%$. The tumor growth suppression rate of AdHTVP2G5-rev-casp3 was $81.52 \%$, significantly higher than that of AdHT-rev-casp3 (54.94\%) or Ad-rev-casp3 (21.35\%). AdHTVP2G5-rev-casp3 significantly improved the survival of tumor-bearing mice with little liver damage, with a mean survival of $258 \pm 28$ days.
\end{abstract}

Correspondence to: Dr Zhijun Xia, Department of Obstetrics and Gynecology, Shengjing Hospital, China Medical University. No. 36, Sanhao Street, Shenyang 110004, P.R. China

E-mail: ssyue@sohu.com

Key words: human telomerase reverse transcriptase, promoter, TSTA, caspase-3, human ovarian carcinoma
These results showed that AdHTVP2G5-rev-casp3 caused effective apoptosis with significant tumor selectivity, strongly suppressed tumor growth and improved mouse survival with little liver toxicity. It can be a potent therapeutic agent for tumor targeted treatment of ovarian cancer.

\section{Introduction}

Apoptosis, or programmed cell death, is a physical form of cell death that plays a central role in tissue homeostasis and in the regulation of the cell suicide program. Apoptosis is necessary to prevent cancer and its induction is a promising approach for cancer gene therapy (1). Several proteins that control programmed cell death in cancer cells have been identified (1). Caspase- 3 is one of the members of the family of cysteine proteases which play a major role in the transduction of apoptotic signals and the execution of apoptosis in mammalian cells (2). There has been considerable interest in caspase-3 as a potent tool for cancer gene therapy, yet ectopic overexpression of the wild-type caspase-3 precursor in cells does not induce apoptosis, due to their inability to undergo autocatalytic activation. We constructed constitutively active recombinant caspase-3 precursors (rev-caspase-3) by making their small subunits preceding their large subunits, as described by Srinivasula et al (3) and Song and Shen (4). Unlike their wild-type counterparts, rev-caspase-3 is capable of autocatalytic processing in an in vitro translation reaction, suggesting that they are catalytically active. Our data demonstrated that overexpression of rev-caspase- 3 can significantly induce apoptotic cell death in both tumor and normal cells in vitro and in vivo (4). This result is in accordance with that of Yang et al (5).

To prevent the toxicity of apoptotic genes in normal cells, targeted expression of therapeutic proteins within the tumor mass is desired. Tumor-restricted gene expression through transcriptional targeting is an attractive approach to reduce toxicity and potentially increase the efficacy of cancer gene therapy. Human telomerase reverse transcriptase (hTERT) is the catalytic subunit of human telomerase and is highly active in immortalized cell lines and in over $85 \%$ of human cancers, but is inactive in most somatic cells $(6,7)$. It has been shown 
that the hTERT promoter (hTERTp) is useful for targeted transgene expression in human ovarian cancer cells (8). However, hTERTp is relatively weak resulting in insufficient transgene expression levels. To overcome this major limitation, we set out to generate an amplified hTERTp for efficient transcriptional targeting of rev-caspase-3 gene expression. We constructed a two-step transcription amplification (TSTA) system by using hTERTp (named the 'hTERTp-TSTA' system) to drive a chimeric transcription factor consisting of the powerful herpes simplex virus VP16 transcriptional activation domain fused to the DNA-binding domain of the yeast protein GAL4, which then binds to the GAL4-binding sites upstream of a minimal promoter to activate rev-caspase-3 gene expression. Our in vitro and in vivo data comparing adenovirus-mediated rev-caspase-3 gene therapy utilizing the binary promoter system vs. a cytomeglovirus promoter (CMVp) demonstrated strong hTERT-restricted antitumor activity with significant reduction in liver toxicity.

\section{Materials and methods}

Cell culture. Human ovarian adenocarcinoma cell lines AO, OVCAR3 and HO8910 were developed in our laboratory and maintained in RPMI-1640 medium (Gibco, Grand Island, NY, USA) supplemented with $10 \%$ heat-inactivated fetal bovine serum (FBS) at $37^{\circ} \mathrm{C}$ with $5 \% \mathrm{CO}_{2}$ in air. Human umbilical vein epithelial cells (HUVECs; CBI, USA) were maintained in growth medium of M200 (CBI, USA) supplemented with $2 \%$ low serum growth supplement (LSGS; CBI, USA). All of the cells were incubated at $37^{\circ} \mathrm{C}$ with $5 \% \mathrm{CO}_{2}$ in air.

Recombinant adenoviral vectors. AdHT-rev-casp3 and Ad-rev-casp3 are recombinant adenoviruses expressing rev-caspase-3 driven by hTERTp and CMVp, respectively, which were prepared using the methods described previously (9). AdHTVP2G5-EGFP, AdHT-EGFP and Ad-EGFP, which express enhanced green fluorescent protein (EGFP) driven by the hTERTp-TSTA system, hTERTp and CMVp, respectively, were successfully constructed in our laboratory previously.

The vector of pHTVP2G5-rev-casp3, a bicistronic adenoviral vector that expresses rev-caspase-3, driven by the hTERTp-TSTA system via the GAL4 gene regulatory system was constructed as follows. pBTdel-279 (kindly denoted by Dr Izumi, National Institute of Environmental Health Science, USA) is a vector that contains the core region of hTERTp from -279 to +5 . pBTdel-279 was cut by KpnI/HindIII, resulting in the hTERTp segment. The resulting segment was then subcloned into the KpnI/HindIII sites of pBluescript SK (+) vector (Takara, Dalian, China), resulting in pBlue-hTERTp. The hTERTp genome was synthesized with following primers of $\mathrm{F}$ and $\mathrm{R}$, using pBlue-hTERTp cDNA as a template: Primer F, 5'-TAA GGT CAC CGG ACC CCC GGG TCC G-3'; and primer R, 5'-GCG TGC TAG CAC TTA GAT CGC AGA TCT CG-3'. The PCR product was ligated with pMD18-T, resulting in pMD18-hTERTp. PBCVP2G5-lucNSN (kindly provided by Professor M. Carey, California University, USA) is a reporter vector containing two components of the TSTA system. pMD18-hTERTp was cut by BstEII and NheI, resulting in hTERTp. The resulting segment was then ligated with
BstEII- and NheI-digested PBCVP2G5-lucNSN, resulting in pHTVP2G5-luc ${ }^{+}$, which expresses luciferase driven by the hTERTp-TSTA system. pHTVP2G5-luc ${ }^{+}$was cut by $\mathrm{XbaI} / \mathrm{SalI}$, resulting in the segment of pA ( 268 bp). pMD18T-SS-LS, which is the pMD18-T vector (Takara) containing rev-caspase-3, was constructed by our laboratory using a method described previously (3). The XbaI/SalI fragments of pA and pMD18-T-SS-LS were ligated and then cut by $S s p \mathrm{I}$ and $S a l \mathrm{I}$, resulting in rev-caspase- $3^{+} \mathrm{pA}$. The $N$ coI-digested and blunted pHTVP2G5-luc ${ }^{+}$was cut by SalI and then ligated with rev-caspase- $3^{+} \mathrm{pA}$, resulting in pHTVP2G5-rev-casp3, which expresses rev-caspase-3 driven by the hTERTp-TSTA system. The insertion was confirmed by restriction enzyme analysis and DNA sequencing analysis. The XbaINotI fragment of pDC316 (Vector Gene Technology, Beijing, China) and pHTVP2G5-rev-casp3 were blunted, cut by SalI and then ligated, resulting in pDCHTVP2G5-rev-casp3, a recombinant adenoviral vector expressing rev-caspase-3 driven by the hTERTp-TSTA system. Briefly, this vector contains two expression cassettes: one for rev-caspase-3, whose gene is driven by a synthetic, minimal promoter composed of five sets of GAL4 binding sites and a TATA sequence (GT promoter), the other for GAL4/VP16, a transactivator whose gene is driven by hTERTp.

pDCHTVP2G5-rev-casp3 was cotransfected into 293 cells with pBHGloxdelE13cre plasmids (Vector Gene Technology) using a lipid-based transfection method (Invitrogen, USA) to generate AdHTVP2G5-rev-casp3. Each recombinant adenovirus was isolated from a single positive plaque and passed through three rounds of plaque purification and subsequently confirmed by PCR and restriction enzyme analysis. All viruses were propagated in 293 cells, purified by ultracentrifugation in a cesium chloride gradient and subjected to dialysis. Viral titer was measured by a standard plaque assay using 293 cells and by absorbance of the dissociated virus at $A_{260 \mathrm{~nm}}$. Titers for subsequent experiments were viral particles (v.p.) per milliliter determined by $A_{260 \mathrm{~nm}}$. Viral preparations had a ratio of v.p. to plaque-forming units (pfu) 100:1. The cells were infected by adenoviral vectors at $37^{\circ} \mathrm{C}$ for $2 \mathrm{~h}$.

EGFP expression assays. Cellular EGFP expression was quantitatively examined by FACS analysis and visualized using fluorescence microscopy. Cells were collected at $48 \mathrm{~h}$ after virus infection. Cells $\left(1 \times 10^{6}\right)$ were illuminated at $488 \mathrm{~nm}$ and fluorescence was detected in the FITC channel. Non-specific fluorescence was detected using a 575/30-nm emission filter in the PI channel. EGFP fluorescence is the mean fluorescence signal in $\mathrm{EGFP}^{+}$cells in relative light units (RLUs) after subtraction of background fluorescence. An inverted-system fluorescence microscope (Olympus, Japan) was used for detection of EGFP expression in cell monolayer.

Cell viability assays. Cells were seeded at a density of $1.7 \times 10^{4}$ cells per well on 96-well plates and after an overnight incubation were treated with AdHTVP2G5-rev-casp3, AdHT-rev-casp3, Ad-rev-casp3 or AdHTVP2G5-EGFP at the indicated doses. Cell viability was assessed using the Dojindo Cell Counting Kit-8 (Dojindo Laboratories, Gaithersburg, MD, USA) according to the supplier's recommendations. Absorbance was read at $450 \mathrm{~nm}$, and cell viability was expressed as the 
percentage of viable cells relative to untreated cells. All experiments were performed in triplicate and at least three times independently. Ninety-six hours later, the surviving cells were counted. Results are presented as a percentage of cell survival compared with the cell counts of cultures treated only with PBS.

Cell-cycle analysis. Treated cells were washed once with phosphate-buffered saline (PBS), trypsinized and washed again in PBS with 2\% FBS and then fixed in ice-cold ethanol for at least $1 \mathrm{~h}$ at $-20^{\circ} \mathrm{C}$. After washing with PBS, the cells were stained with propidium iodide $(30 \mu \mathrm{g} / \mathrm{ml})$ and treated with RNase $(0.6 \mathrm{mg} / \mathrm{ml})$ in PBS plus $0.5 \%(\mathrm{v} / \mathrm{v})$ Tween-20 and $2 \%$ FBS. Stained cells were analyzed on a FACSCalibur flow cytometer (BD Bioscience) using CellQuest software, and the ModFit program (Verity Software House Inc., Topsham, ME, USA) was used to analyze the cell cycle profiles.

Real-time PCR. Total cellular RNA was isolated using TRIzol reagent (Invitrogen, Carlsbad, CA, USA). First-strand cDNA synthesis was performed using reverse transcriptase (Invitrogen) by incubation at $25^{\circ} \mathrm{C}$ for $10 \mathrm{~min}, 37^{\circ} \mathrm{C}$ for $60 \mathrm{~min}$ and $95^{\circ} \mathrm{C}$ for $5 \mathrm{~min}$. The sequences of the primers used for real-time PCR were as follows: hTERT, 5'-CGG AAG AGT GTC TGG AGC AA-3' (sense) and 5'-GGA TGA AGC GGA GTC TGG A-3' (antisense); active caspase-3, 5'-CCATGCTGA AACAGTATGCCG-3' (sense) and 5'-TTCCAGAGTCCA TTGATTCGCT-3' (antisense); GAPDH, 5'-ACCACAGTCCA TGCCATCAC-3' (sense) and 5'-TCCACCACCCTGTT GCTGTA-3 (antisense). Amplification was performed in a 25- $\mu 1$ reaction containing $2 \mu \mathrm{l}$ sample cDNA, 0.4X TaqMan Universal PCR Master Mix (Applied Biosystems, USA), $120 \mathrm{nM}$ of each primer and $1 \mathrm{nM}$ DNA probe. The PCR was run at $94^{\circ} \mathrm{C}$ for $2 \mathrm{~min}$ followed by 40 cycles of $94^{\circ} \mathrm{C}$ for $5 \mathrm{sec}$, $62^{\circ} \mathrm{C}$ for $10 \mathrm{sec}$ and $60^{\circ} \mathrm{C}$ for $30 \mathrm{sec}$. Amplification was performed according to the manufacturer's specifications.

Immunoblotting studies. To detect expression of exogenous active caspase- 3 and induction of apoptosis in AO cells and HUVECs, $1 \times 10^{6}$ cells were plated onto $6-\mathrm{cm}$ dishes and infected with AdHTVP2G5-rev-casp3 at a multiplicity of infection (MOI) of 70. Cell lysates were prepared as previously described. Immunoblotting procedure was carried out as previously described (10). AdHT-rev-casp3 and Ad-rev-casp3 at the same doses were used as controls. The following antibodies were used for immunoblotting: anti-PARP antibody and anti-caspase-3 antibody (Cell Signaling Technology, Danvers, Inc., MA, USA).

Xenograft studies. The study protocol was approved by the local institution review boards at the affiliated institutions of the authors. Female mice, 4-6 weeks old and weighing 16-20 g (Animal Research Institution of Chinese Academy of Medical Sciences, Beijing, China), were used for the experiment. All animals were cared for according to the guidelines for the Care and Use of Laboratory Animals and the Institutional Guidelines of the Chinese Academy of Medical Sciences. For the subcutaneous tumor model, $1 \times 10^{7} \mathrm{AO}$ cells in $0.2 \mathrm{ml}$ PBS were inoculated subcutaneously into the dorsal flank of nude mice. When the tumor reached a volume of $\sim 150 \mathrm{~mm}^{3}$, the mice received an intratumoral injection of PBS, AdHTVP2G5-rev-casp3 [2.5×10 ${ }^{9}$ tissue culture infectious dose $50\left(\mathrm{TCID}_{50}\right) /$ tumor], AdHT-rev-casp3 (2.5x10 ${ }^{9} \mathrm{TCID}_{50} /$ tumor), Ad-rev-casp3 $\left(2.5 \times 10^{9} \mathrm{TCID}_{50} /\right.$ tumor $)$ or AdHTVP2G5-EGFP $\left(2.5 \times 10^{9} \mathrm{TCID}_{50} /\right.$ tumor $)$. Three intratumoral injections were given every 10 days, and 5 mice from each group were followed up once every 3 days to measure tumor size by calipers. Tumor volumes were calculated using the formula: $\mathrm{a} \mathrm{x}^{2} \times 0.5$, where $a$ and $b$ represent the larger and smaller diameters, respectively. Mice were sacrificed according to the institutional guidelines when the tumor with PBS (negative control) treatment reached $2000 \mathrm{~mm} 3$ in volume.

For the peritoneal tumor model, $1 \times 10^{7} \mathrm{AO}$ cells resuspended in $0.5 \mathrm{ml}$ of $\mathrm{PBS}$ were injected intraperitoneally into nude mice. Subsequently, mice were weighed once every two days. Twenty-one days after inoculation, the mice received an intraperitoneal injection of PBS, AdHTVP2G5-rev-casp3 (2.5x10 ${ }^{9} \mathrm{TCID}_{50} /$ tumor $)$, AdHT-rev-casp3 (2.5x $10^{9} \mathrm{TCID}_{50} /$ tumor $)$, Ad-rev-

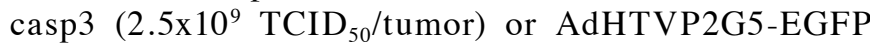
$\left(2.5 \times 10^{9} \mathrm{TCID}_{50} /\right.$ tumor). Three intraperitoneal injections were given every 10 days. Three mice from each group were sacrificed according to the institutional guidelines $72 \mathrm{~h}$ after the first injection to detect the expression of EGFP or active caspase- 3 by fluorescence microscopy or RT-PCR, in the tumor and liver tissues, respectively. Survival was defined as the study endpoint. Histopathological changes in the liver, spleen, intestine, lung, kidney, ovary, pancreas and heart were examined, and serum contents of alanine transaminase (ALT) and aspartate transaminase (AST) were determined as described previously (12). Blood samples were collected via the tail vein on day 1 and 14 to monitor liver damage and specifically, the serum levels of AST and ALT in each group.

Statistical analysis. Statistical differences among the treatment groups were assessed by ANOVA using SPSS16 software program. A value $\mathrm{P}<0.05$ was considered to indicate a statistically significant result. Additionally, the survival data were summarized and plotted using the Kaplan-Meier method, and survival curves were compared using the log-rank test.

\section{Results}

Selective strong activity of the hTERTp-TSTA system in hTERT-positive cells. We constructed recombinant adenoviral vector AdHTVP2G5-EGFP which contains two expression cassettes and expresses EGFP driven by the hTERTp-TSTA system. One cassette expresses the EGFP gene driven by the GAL4/TATA (GT) promoter, while the other expresses the GAL4/VP16 fusion gene driven by the hTERTp. To assess the activity and selectivity of the hTERTp-TSTA system in various cells, we infected hTERT-positive ovarian cancer AO, OVCAR3 and HO8910 cells and hTERT-negative normal cell HUVECs with AdHTVP2G5-EGFP, AdHT-EGFP or Ad-EGFP at an MOI of 70 for $2 \mathrm{~h}$. Cells were harvested $48 \mathrm{~h}$ after infection, and EGFP expression was analyzed by fluorescence-activated cell sorting (FACS).

We found that the hTERTp-TSTA system showed the strongest activity when compared with hTERTp and CMVp, although these three promoter systems were all active in the 
A

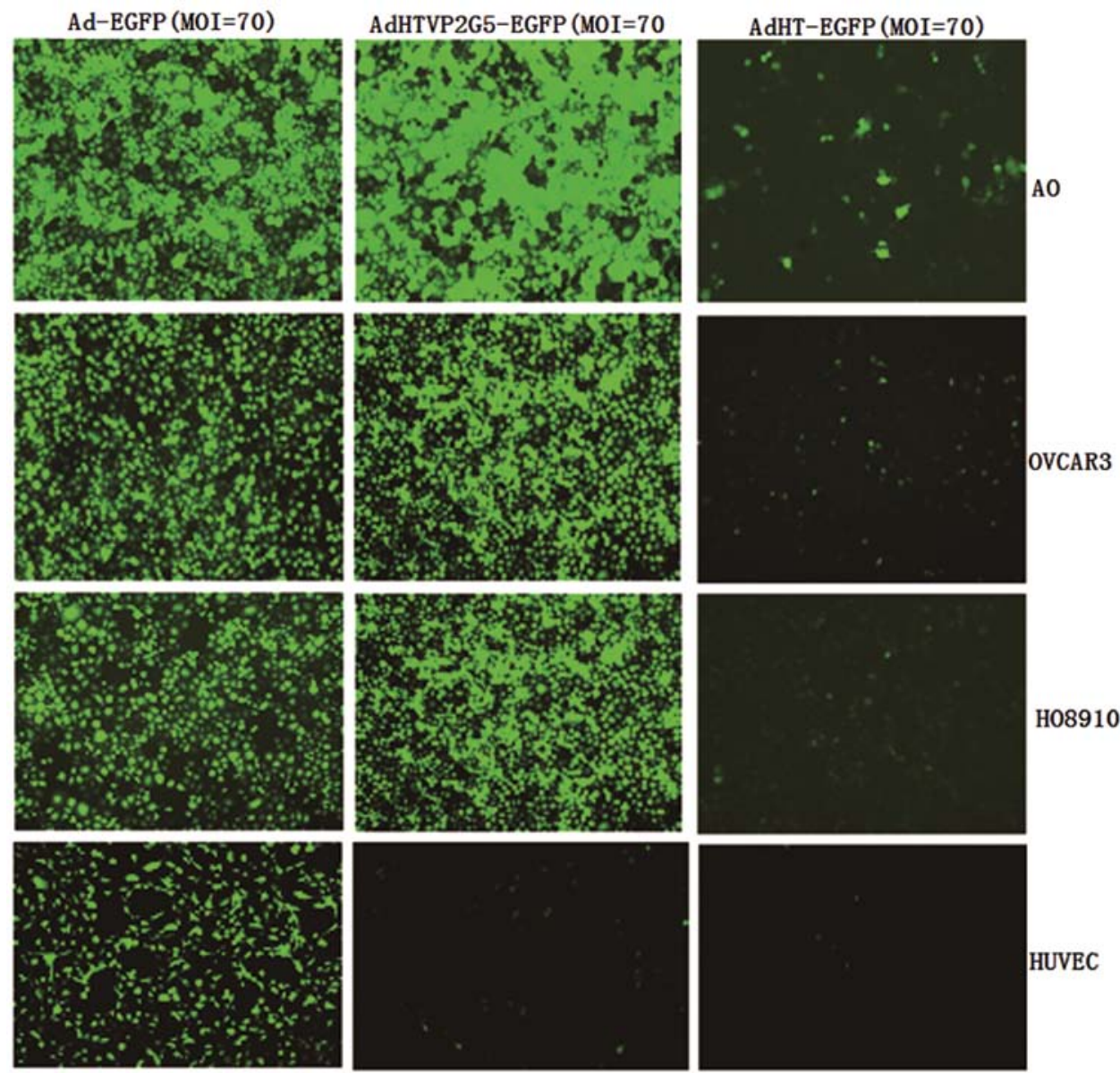

B

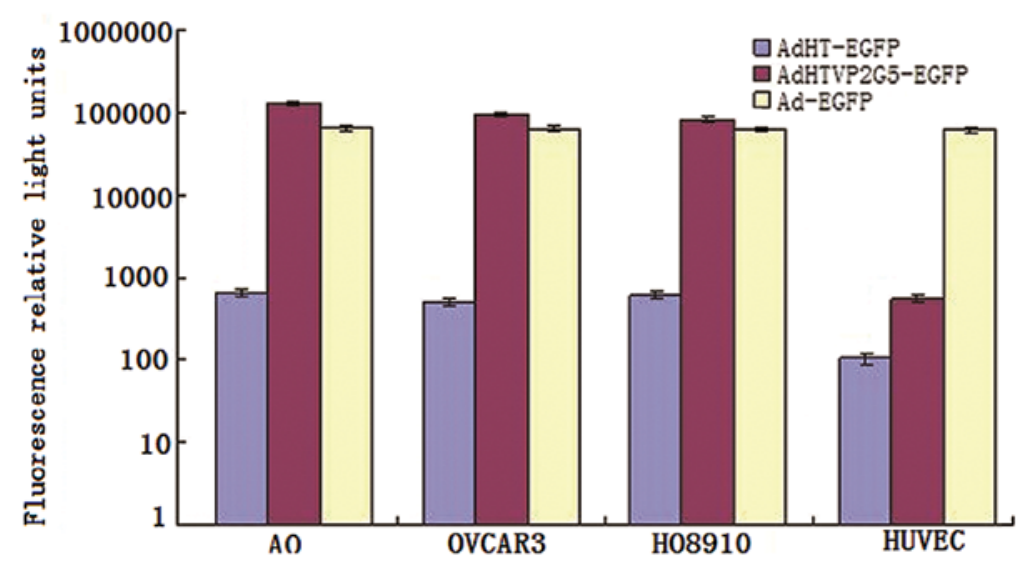

Figure 1. In vitro analysis of hTERTp-TSTA system activities. Cells were treated with the same doses (MOI=70) of AdHTVP2G5-EGFP, AdHT-EGFP and Ad-EGFP. (A) Expression of EGFP was revealed under a fluorescencet microscope. The name of each cell line is indicated to the left of the panels and the treatments are indicated at the top of panels. (B) Samples (1x10 cells) were analyzed by FACScan. EGFP fluorescence was expressed as the mean fluorescence signal in $\mathrm{EGFP}^{+}$cells in RLUs after subtraction of background fluorescence. Presented are mean values of three independent experiments, each carried out in triplicate. hTERTp, human telomerase reverse transcriptase promoter; EGFP, enhanced green fluorescent protein.

hTERT-positive cancer cells. The RLUs in the AO, OVCAR3 and HO8910 cells infected with AdHTVP2G5-EGFP were $12,800,95,210$ and 83,500, which were 203-, 193- and 140 -fold higher than the RLUs in the cells infected by AdHTEGFP and were as 2.0-, 1.5- and 1.3-fold in the cells infected by Ad-EGFP, respectively. In contrast, AdHTVP2G5-EGFP demonstrated a background level of EGFP expression (RLUs of 345) in the hTERT-negative HUVECs, being 180-fold lower than that of Ad-EGFP (RLUs of 62,200) and showing no significant difference with that of AdHT-EGFP (RLUs of 103) (Fig. 1A and B).

AdHTVP2G5-rev-casp3 markedly suppresses the survival of $A O$ cells with tumor selectivity in vitro. We infected $\mathrm{AO}$ cells with AdHTVP2G5-rev-casp3, AdHT-rev-casp3 or Ad-rev-casp3 at an MOI of 5 to 200 and $96 \mathrm{~h}$ later examined the viability of these cells. The viability assays using the CCK-8 method showed that AdHTVP2G5-rev-casp3 signifi- 
A

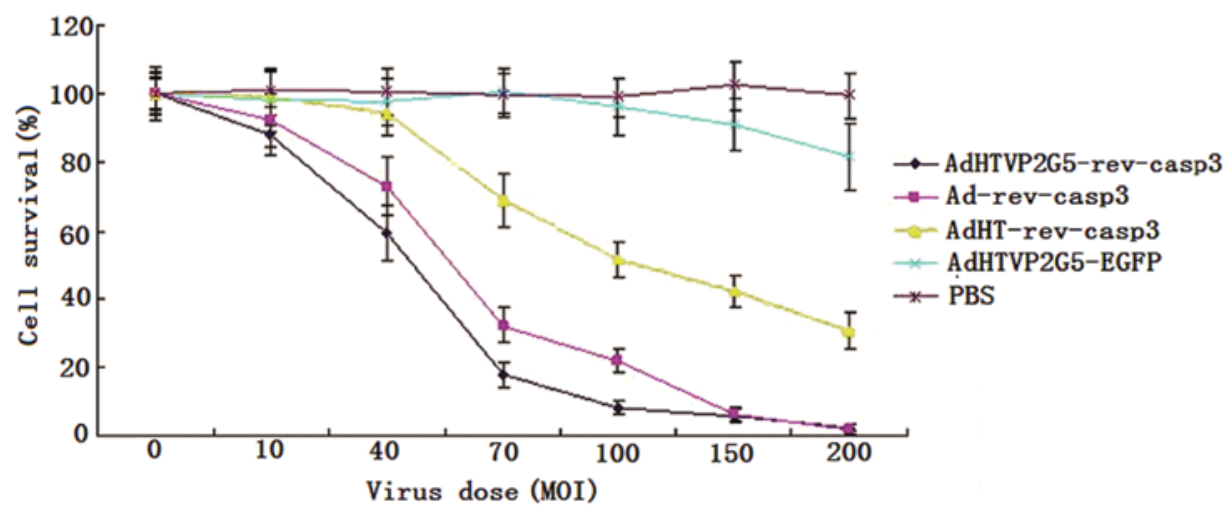

B

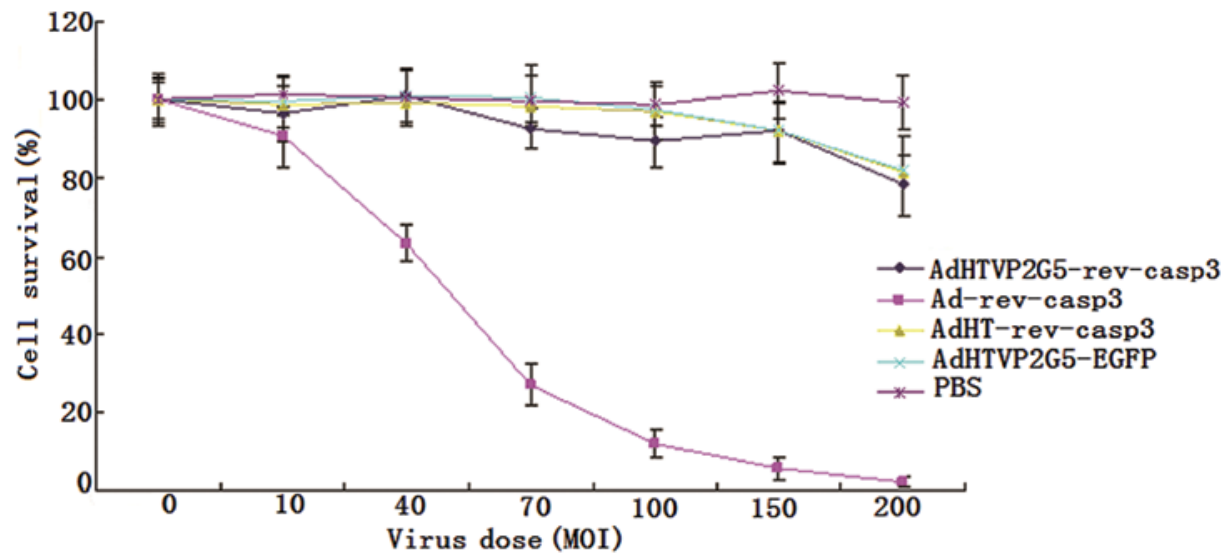

C

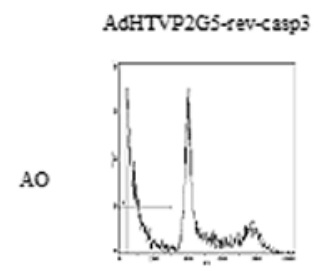

$42 \%$

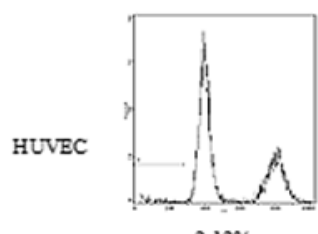

$2.12 \%$

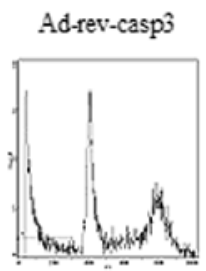

$35.82 \%$

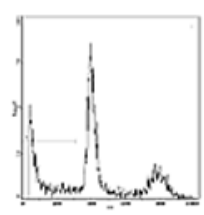

$38.12 \%$

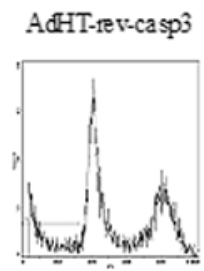

$25.97 \%$

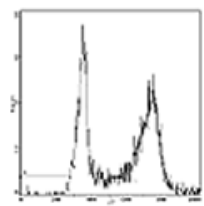

$1.68 \%$
AdHTVP2G5-EGFP

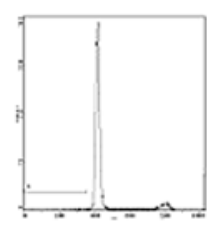

$0.94 \%$

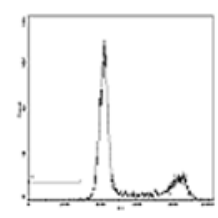

0.5496

Figure 2. Efficacy and selectivity of cell killing activity of AdHTVP2G5-rev-casp3, AdHT-rev-casp3 and Ad-rev-casp3 in vitro. (A and B) Cell viability determined by Counting Kit-8 assay. AO cells (hTERT-positive) and HUVECs (hTERT-negative) were treated by various vectors using different MOIs as indicated. The standard deviation for triplicate wells was <10\%. (C) Flow cytometric analysis of apoptotic (sub-G1) AO and HUVECs. The name of each cell line is indicated to the left of the panels and the treatments are indicated at the top of panels.

cantly suppressed the survival of AO cells in a dose-dependent manner with a viability rate of $17.8 \pm 3.5 \%$ at an MOI of 70 and $8.1 \pm 1.9 \%$ at an MOI of 100 (Fig. 2A), which was markedly lower than that of AO cells treated with Ad-rev-casp3 $(32.3 \pm 5.3 \%$ at an MOI of 70 and $21.8 \pm 3.4 \%$ at an MOI of 100$)$ and AdHT-rev-casp3 (69.2 $\pm 7.8 \%$ at an MOI of 70 and $51.6 \pm 5.3 \%$ at an MOI of 100), respectively. Flow cytometry additionally showed that the sub-G1 fraction increased to 42, 35.82 and $25.97 \%$ in the AO cells treated with AdHTVP2G5-rev-casp3, Ad-rev-casp3 and AdHT-rev-casp3, respectively, at an MOI of 70, which was markedly higher than that of AO cells treated with AdHTVP2G5-EGFP controls (0.94\%, P<0.05).
We next examined the effect of AdHTVP2G5-rev-casp3, AdHT-rev-casp3 or Ad-rev-casp3 on the proliferation of HUVECs. A significant difference was detected in HUVEC viability following three reconstructive adenoviruses, respectively. AdHTVP2G5-rev-casp3 induced little HUVEC death with the cell viability rate of $92.7 \pm 5.2$ and $89.2 \pm 6.3 \%$ at an MOI of 70 and 100, respectively, which showed no significant difference with that induced by AdHT-rev-casp3 (98.5 \pm 6.9 and $96.9 \pm 7.7 \%$, respectively). In contrast, Ad-rev-casp3 induced significant cell death in the HUVECs with a cell viability rate of $27.1 \pm 5.4$ (MOI of 70 ) and $11.9 \pm 3.6 \%$ (MOI of 100), respectively, which was similar to that in the AO 
A

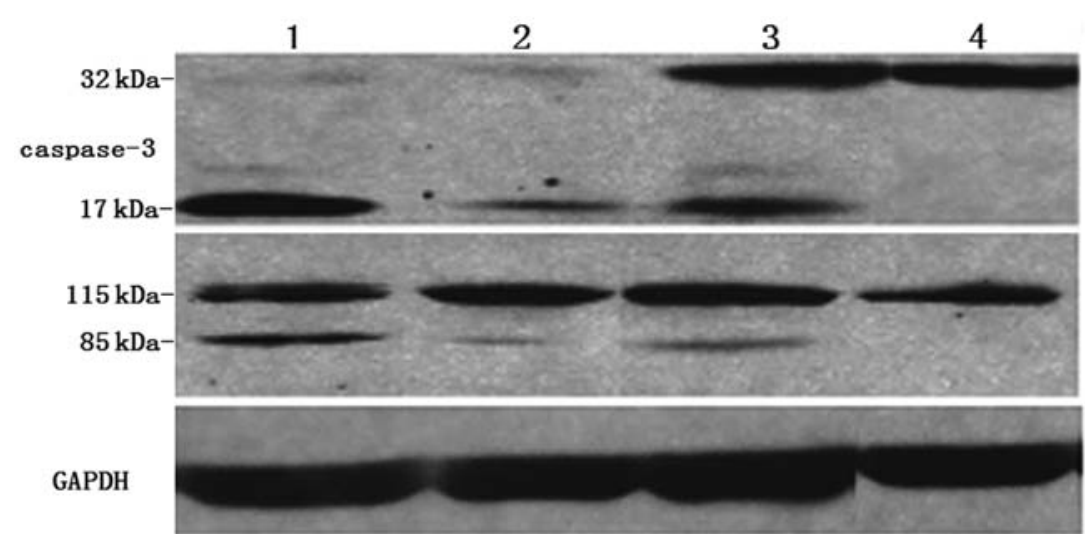

B

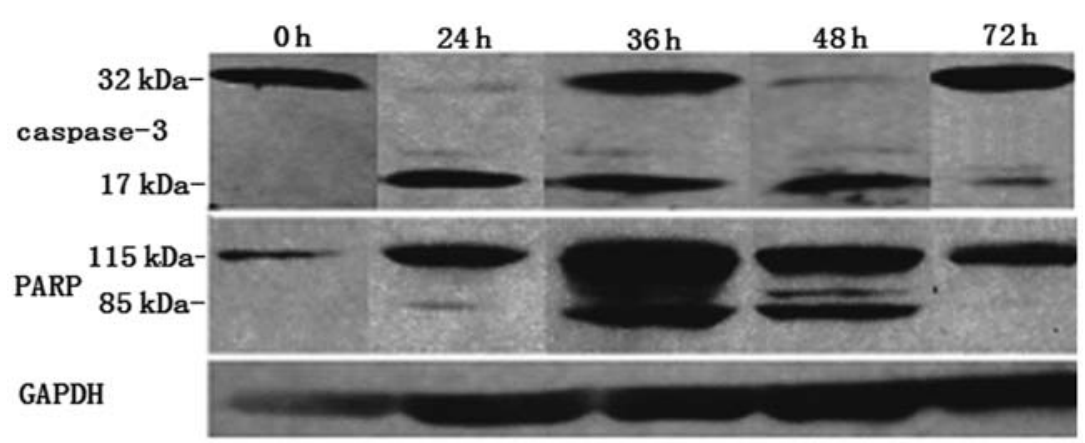

Figure 3. Western blot analysis of active caspase-3 expression and apoptosis induction in AO and HUVEC cells. (A) Caspase-3 activation and PARP cleavage expressions in AO cells $30 \mathrm{~h}$ after infection of rev-caspase-3 driven by various promoter systems. Lane 1, AdHTVP2G5-rev-casp3; lane 2, AdHT-rev-casp3; lane 3, Ad-rev-casp3; lane 4, negtive control;.(B) Caspase-3 activation and PARP cleavage expression in AO cells 0-72 hafter infection of AdHTVP2G5-rev-casp3 $(\mathrm{MOI}=70)$.

cells (Fig. 2B). Flow cytometry further showed that at an MOI of 70, AdHTVP2G5-rev-casp3 and AdHT-rev-casp3 did not induce significant apoptosis with an apoptotic rate of only 2.1 and $1.7 \%$ in the HUVECs, respectively, whereas Ad-rev-casp3 induced significant apoptosis with an apoptotic rate of $38.1 \%$ in the HUVECs, similar to that in the AO cells (Fig. 2C).

AdHTVP2G5-rev-casp3 causes significant apoptotic activities in AO cells with significant tumor selectivity. We further examined the levels of active caspase- 3 in AO cells and HUVECs treated with various adenoviral vectors at an MOI of 70, $48 \mathrm{~h}$ after viral infections by RT-PCR. We found that the mRNA transcript levels of active caspase- 3 in the AO cells treated with AdHTVP2G5-rev-casp3 (9.44 \pm 1.36$)$ was significantly higher than that with Ad-rev-casp3 $(8.19 \pm 1.72)$, AdHT-rev-casp3 $(4.47 \pm 1.9)$ and AdHTVP2G5-EGFP $(1.17 \pm 1.5)(\mathrm{P}<0.05)$, respectively, while no apparent active caspase-3 mRNA expression was found in the HUVECs treated with AdHTVP2G5-rev-casp3 $(1.95 \pm 1.28)$ and AdHT-rev-casp3 (1.55 \pm 1.41$)$ or AdHTVP2G5-EGFP control. By contrast, Ad-rev-casp3 caused a significant increase in active caspase- 3 mRNA levels in both AO cells $(8.66 \pm 1.8)$ and the HUVECs $(7.93 \pm 2.1)$.

Our immunoblotting analysis further revealed that the AdHTVP2G5-rev-casp3-, AdHT-rev-casp3- and Ad-rev-casp3-treated AO cells expressed significant levels of p17 and p85 proteins. Furthermore, the levels of p17 and p85 proteins in the AdHTVP2G5-rev-casp3-treated cells were significantly higher than levels in the Ad-rev-casp3-or
AdHT-rev-casp3-treated cells $30 \mathrm{~h}$ after the infections. In contrast, in HUVECs, only Ad-rev-casp3-treated cells significantly expressed $\mathrm{p} 17$ and $\mathrm{p} 85$ proteins, while AdHTVP2G5-rev-casp3 and AdHT-rev-casp3 treatments did not result in expression of p17 and p85 in the cells (Fig. 3A). These findings further confirmed that AdHTVP2G5-rev-casp3 caused selective strong apoptotic activities in the AO cells, not in the HUVECs.

p17 proteins became evident as early as $24 \mathrm{~h}$ after AdHTVP2G5-rev-casp3 infection of AO cells and became stronger at $36-48 \mathrm{~h}$ after the infection. In addition, $\mathrm{p} 85$ proteins were detected as early as $24 \mathrm{~h}$ and became evident at $36-48 \mathrm{~h}$ after the infection. Protein expression of both p17 and p85 became weak at $72 \mathrm{~h}$ after infection (Fig. 3B).

hTERTp-TSTA system drives tumor-specific EGFP and rev-caspase-3 gene expression in vivo. We gave mice bearing abdominal tumors derived from AO cells an intraperitoneal injection of AdHTVP2G5-EGFP, AdHT-EGFP or Ad-EGFP at a single dose of $2.5 \times 10^{9} \mathrm{TCID}_{50} /$ mouse. We sacrificed the mice $72 \mathrm{~h}$ after injection and collected the tumor and liver tissues for fluorescence detection to assess EGFP expression. We found that all AdHTVP2G5-EGFP, AdHT-EGFP and Ad-EGFP caused EGFP expression in tumor tissues. In comparison, only Ad-EGFP induced strong EGFP expression in the liver, while AdHTVP2G5-EGFP and AdHT-EGFP induced very weak EGFP expression (Fig. 4). These results demonstrate that hTERT-TSTAp is highly active in tumors, but quiescent in normal liver in vivo. We observed similar selective expression 

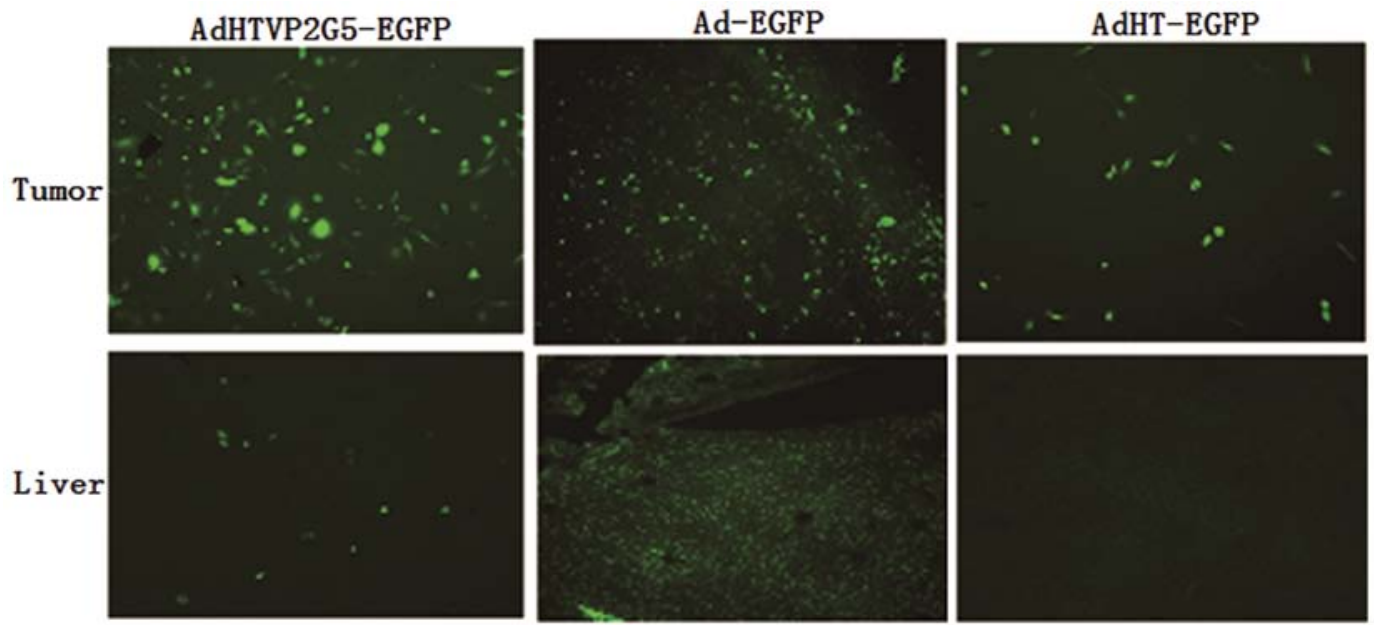

Figure 4. Analysis of EGFP gene expression by observation of frozen section under a fluorescence microscope. Treatments are indicated above each panel. Tumor and liver samples were collected $72 \mathrm{~h}$ after intraperitoneal injection.
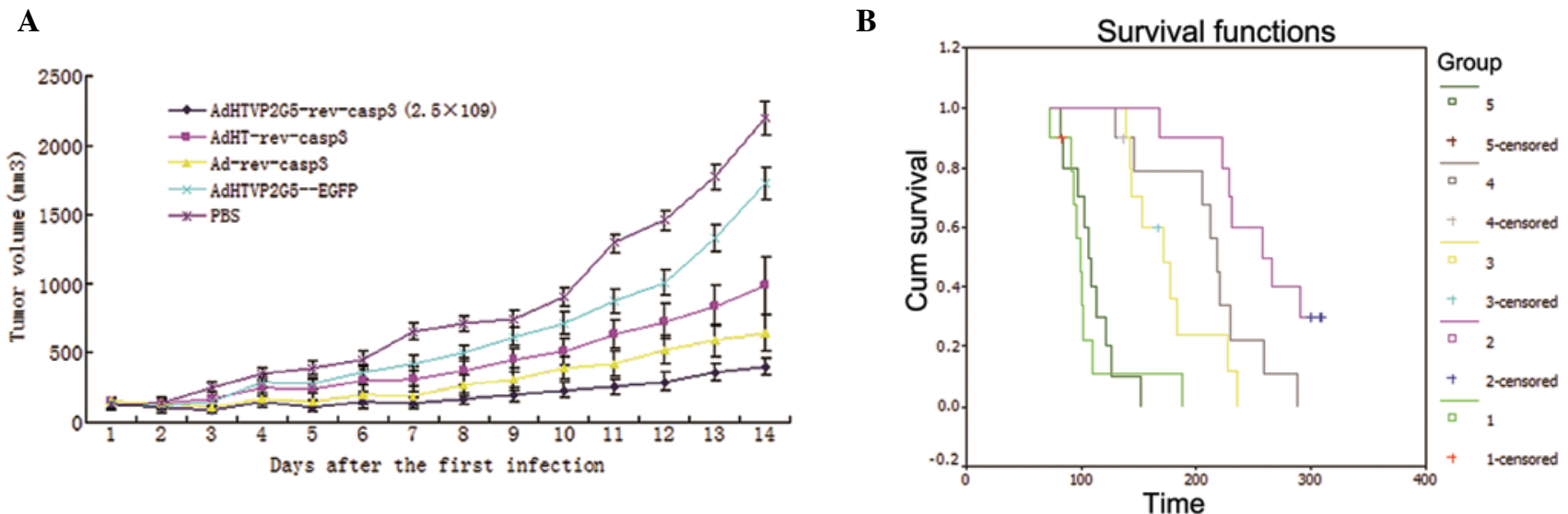

Figure 5. In vivo antitumor activity. (A) Subcutaneous tumors derived from AO cells were treated with various vectors. Tumors volumns were monitored over time (days) after inoculation of tumor cells. Values represent the mean of 5 mice per group; (B) Survival curves for animals bearing abdominally spread AO tumors. The animals received the treatments as shown: 1: PBS; 2: AdHTVP2G5-rev-casp3; 3: AdHT-rev-casp3; 4: Ad-rev-casp3; 5: AdHTVP2G5-EGFP. Survival was then monitored: the survival duration in animals receiving treatment using AdHTVP2G5-rev-casp3, AdHT-rev-casp3 or Ad-rev-casp3 were significantly differently from those that received control treatments $(\mathrm{P}<0.01)$. Furthermore, AdHTVP2G5-rev-casp3 treatments prolonged survival significantly when compared with treatment using AdHT-rev-casp3or Ad-rev-casp3 $(\mathrm{P}<0.05)$.

of rev-caspase- 3 by RT-PCR when we sacrificed the mice $72 \mathrm{~h}$ after injection with AdHTVP2G5-rev-casp3, AdHT-rev-casp3 or Ad-rev-casp3 and collected their tumor and liver tissue for caspase-3 expression. AdHTVP2G5-rev-casp3 induced strong caspase-3 expression in the tumors, while it did not induce detectable transgene expression in the liver.

AdHTVP2G5-rev-casp3 markedly suppresses tumor growth and extends the survival of tumor xenograft-bearing mice. To evaluate the in vivo efficacy of AdHTVP2G5-rev-casp3, we established a mouse xenograft model by inoculating AO cells subcutaneously in nude mice and we treated the mice with AdHTVP2G5-rev-casp3, AdHT-rev-casp3 or Ad-rev-casp3, respectively. Fifty-three days after treatment, the tumor volume was $406 \pm 61,990 \pm 214$ and $645 \pm 132 \mathrm{~mm}^{3}$ in mice bearing AO xenografts treated with AdHTVP2G5-rev-casp3, AdHT-rev-casp3 and Ad-rev-casp3, respectively. As shown in Fig. 5A, the tumor growth suppression rates of
AdHTVP2G5-rev-casp3 and Ad-rev-casp3 were 81.52 and $70.64 \%$, respectively, significantly higher than that of AdHT-rev-casp3 (54.94\%) or AdHTVP2G5-EGFP (21.35\%) at the endpoint of the study (Fig. 5A).

We next tested the effect of AdHTVP2G5-rev-casp3 in vivo in an abdominally spread tumor model of $\mathrm{AO}$ cells. Twenty-one days after inoculation, the mice were randomized to receive intraperitoneal injections of various adenoviral vectors or PBS ( $n=5 /$ group). Survival of mice in each group was recorded. Kaplan-Meier analysis indicated that AdHTVP2G5-rev-casp3, AdHT-rev-casp3 or Ad-rev-casp3 significantly improved the survival of mice receiving an intraperitoneal inoculation of AO cells compared with control mice treated with PBS $(\mathrm{P}<0.05)($ Fig. 5B). The mean survival of mice treated with AdHTVP2G5-rev-casp3 was $259 \pm 14$ days with a median survival of $258 \pm 28$ days. Furthermore, AdHTVP2G5-rev-casp3 treatments prolonged survival significantly when compared with treatment using AdHT-rev-casp3 
A

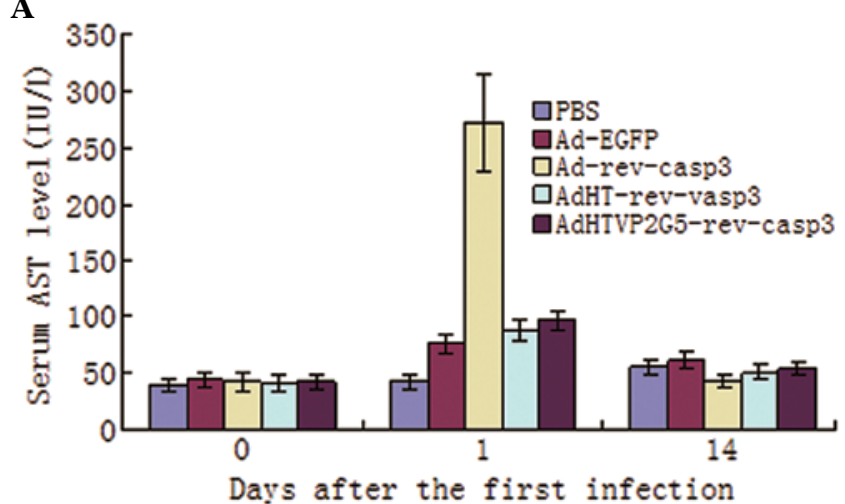

B

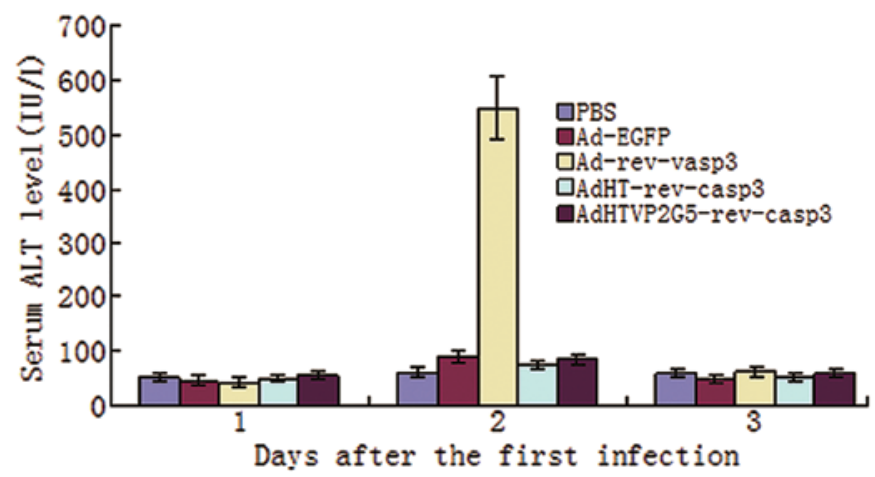

Figure 6. Serum (A) AST and (B) ALT levels. Serum samples were collected before treatment started (day 0) and 1 (day 1) and 14 days (day 14) after the last treatment.

Table I. Mean and median survival of mice receiving the various adenoviral vectors $(\mathrm{n}=10)$.

\begin{tabular}{lllc}
\hline Group & $\begin{array}{c}\text { Mean } \\
\text { (days) }\end{array}$ & N & $\begin{array}{c}\text { Median } \\
\text { (days) }\end{array}$ \\
\hline PBS & $106 \pm 11$ & 10 & $99 \pm 6$ \\
AdHTVP2G5-EGFP & $109 \pm 7$ & 10 & \\
AdHTVP2G5-rev-casp3 & $259 \pm 14^{\mathrm{a}}$ & 10 & $258 \pm 28$ \\
AdHT-rev-casp3 & $177 \pm 12^{\mathrm{b}}$ & 10 & $171 \pm 16$ \\
Ad-rev-casp3 & $213 \pm 16^{\mathrm{c}}$ & 10 & $218 \pm 7$ \\
\hline
\end{tabular}

${ }^{\mathrm{a}} \mathrm{P}<0.05,{ }^{\mathrm{b}} \mathrm{P}<0.05,{ }^{\mathrm{C}} \mathrm{P}<0.05$.

(177 \pm 12 days, $\mathrm{P}<0.05)$ or Ad-rev-casp3 $(213 \pm 16$ days, $\mathrm{P}<0.05)$ (Table I).

Toxicity after intraperitoneal vector administration. We also examined the toxicity profiles of intraperitoneal administration of AdHTVP2G5-rev-casp3, AdHT-rev-casp3 or Ad-rev-casp3. We measured serum ALT and AST levels and found that these levels were only slightly elevated in the mice receiving AdHTVP2G5-rev-casp3 or AdHT-rev-casp3. The AST and ALT levels were significantly lower in mice receiving AdHTVP2G5-rev-casp3 or AdHT-rev-casp3 1 day after treatment than that in mice receiving Ad-rev-casp3 $(\mathrm{P}<0.01)$. On day 14 , no significant difference in AST or ALT was noted among the groups (Fig. 6A and B). We also examined the histopathological changes in the liver, spleen, intestine, lungs, kidneys, ovary and heart and we found no obvious lesions in mice receiving AdHTVP2G5-rev-casp3 or AdHT-rev-casp3.

\section{Discussion}

It has been shown that rev-caspase-3 induces potent apoptosis in human breast cancer, pancreatic cancer and colon cancer cells (2,3-5). In the present study, AdHTVP2G5-rev-casp3 suppressed AO cell growth with a survival rate of $<20 \%$, at $96 \mathrm{~h}$ after its infection at an MOI of 70-100, and the cells displayed typical apoptotic morphology after treatments as detected by electron transmission microscopy. This result demonstrated that the recombinant molecule, rev-caspase-3, has potent activity for inducing apoptosis similar to its wild-type counterparts. The apoptosis induced by rev-caspase- 3 was the most significant at $72 \mathrm{~h}$ after viral infection. We examined the expression of active caspase- 3 and the induction of apoptosis at $24,36,48$ and $72 \mathrm{~h}$ after rev-caspase- 3 transfection. The results showed that active caspase- 3 p17 and PARP cleavage $\mathrm{p} 85$ proteins became evident as early as $24 \mathrm{~h}$ and were strongly expressed at $36-48 \mathrm{~h}$ after the viral infection. Protein expression of both p17 and p 85 became weak at $72 \mathrm{~h}$ after infection, probably due to increased cell death.

The optimal MOI was determined by infecting each cell line with Ad-EGFP and assessing the expression of green fluorescence protein under a fluorescence microscope. The optimal MOI of recombinant adenoviruses expressing rev-caspase-3 was 70-100, at which most of the cells underwent apoptosis. Alternatively, the non-cytotoxic MOI of the viral vectors used (5-20) resulted in cessation of cell growth with significant S-phase delay and retardation in S-phase progression. No significant initial cell apoptosis occurred at this MOI.

It is necessary to restrict the expression of rev-caspase-3 to tumor cells, due to its potent efficacy of inducing apoptosis. Utilization of the hTERT promoter that is predominantly active in tumor cells would be an ideal system to restrict rev-caspase-3 expression. However, in most cancer cells, the hTERTp activity is $>10$-fold lower than that of CMVp (11) and is too weak to achieve sufficient transgene expression. It has been shown that transgene expression from a tumor-specific promoter can be augmented by using a TSTA system (12-14). In the present study, we employed a TSTA system to elevate the activity of the hTERTp up to a 203 -fold range in human ovarian cancer cell lines. In this two-tiered system, the hTERT regulatory region was employed to express the potent synthetic transcription activator, GAL4-VP2, which in turn powerfully activates a minimal TATA promoter to induce transgene expression. In AdHTVP2G5-EGFP, the activator and reporter component were inserted into the adenoviral vector in a divergently linked head-to head configuration. In vitro infection assays of hTERT-positive cells with AdHTVP2G5-EGFP 
confirmed the hTERT-selective expression of this approach. Furthermore, the level of EGFP expression by hTERTp-TSTA was 203 -fold higher than that by hTERTp alone and also compared favorably with CMVp, the strong constitutively active viral promoter.

We then established the binary system expressing rev-caspase- 3 in a single recombinant adenovirus, named AdHTVP2G5-rev-casp3. In vitro cell survival assays in hTERT-positive and hTERT-negative cells confirmed the selectivity of AdHTVP2G5-rev-casp3. Only hTERT-expressing cells were killed by AdHTVP2G5-rev-casp3 as opposed to the non-specific killing of Ad-rev-casp3. Moreover, the cell killing efficacy of AdHTVP2G5-rev-casp3 was comparable to that of Ad-rev-casp3.

The selectivity of the hTERTp-TSTA system in vivo was further confirmed by quantitative analysis of EGFP and active caspae-3 expression. Fluorescence detection and RT-PCR indicated transgene expression only in the area of hTERT-positive tumors with significantly less spread to the adjacent liver tissues than after administration of the universally expressed Ad-rev-casp3. Moreover, AdHTVP2G5-rev-casp3 was more potent in tumor killing than Ad-rev-casp3.

A transcriptionally targeted gene expression approach could reduce the potential side effects of adenovirus-mediated cytotoxic cancer gene therapy (15). After intratumoral injection of adenovirus constitutively expressing luciferase or another reporter gene, leakage of the vector into systemic circulation resulted in transgene expression in the liver (16). From this finding liver toxicity can be anticipated after intratumoral injection of Ad-rev-casp3. The gene-expression targeting approach employed in this study will not alter the in vivo liver distribution observed of Ad5 (17). This preferential adenovirus transduction has contributed to liver toxicity $(18,19)$ due to the innate immune response to viral capsid proteins (20) and cell-mediated immunity against viral gene products $(21,22)$. Utilization of a specific promoter to drive transgene expression was shown to reduce both the immune response against the adenovirus and the associated liver toxicity; however, high viral doses accompanied by severe liver toxicity were necessary to achieve therapeutic effects (23). The potent gene expression mediated by the TSTA system could potentially reduce the amount of vector needed to transduce cancer cells in vivo compared to nonamplified tissue-selective vectors. Reducing the input dosage of Ad has been documented to reduce liver toxicity $(20-22,24)$. Our data suggest that, at an viral dose of $2.5 \times 10^{9} \mathrm{TCID}_{50}$, the AdHTVP2G5-rev-casp3 has the potential to induce tumor killing with reduced liver toxicity. In the present study, the same extent of vector delivery to the liver is expected to occur when hTERTp-TSTA is employed to drive rev-caspase-3; however, rev-caspase-3 expression in the liver is restricted by hTERTp-TSTA and therefore rev-caspase-3-mediated liver toxicity is reduced. The Ad/hTERTp-TSTA system exhibited a lower toxicity profile than the Ad/CMVp system.

In summary, our results provide evidence that the AdHTVP2G5-rev-casp3 is able to treat ovarian cancer with a high degree of efficacy and selectivity. The use of a non-mammalian transcriptional activator ensures that no other endogenous genes are being activated. The TSTA system will undoubtedly profit from the intense investigation of many tissue-specific promoters and may ultimately emerge as a powerful tool for stringent gene expression in a wide variety of tumors.

\section{Acknowledgements}

We thank Professor Izumi for the pBTdel-279 plasmid and useful information, Professor M. Garey for the PBCVP2G5-lucNSN plasmid and Professor K. Wang K help in preparing the adenoviral vectors. This study was supported by NSFC (National Natural Science Foundation of China) grant 30600746 (to Y.S.).

\section{References}

1. Jia LT, Chen SY and Yang AG: Cancer gene therapy targeting cellular apoptosis machinery. Cancer Treat Rev 38: 868-876, 2012.

2. Mazumder S, Plesca D and Almasan A: Caspase-3 activation is a critical determinant of genotoxic stress-induced apoptosis. Methods Mol Biol 414: 13-21, 2008.

3. Srinivasula SM, Ahmad M, MacFarlane M, Luo Z, Huang Z, Fernandes-Alnemri T and Alnemri ES: Generation of constitutively active recombinant caspase- 3 and -6 by rearrangement of their subunits. J Bio Chem 273: 10107-10111, 1998.

4. Song Y and Shen K: Construction of autocatalytic caspase-3 and its effects of inducing apoptosis in human ovarian carcinoma Zhonghua Fu Chan Ke Za Zhi 42: 846-851, 2007 (In Chinese).

5. Yang L, Cao Z, Yan H and Wood WC: Coexistence of high levels of apoptotic signaling and inhibitor of apoptotic proteins in human tumor cells: implication for cancer specific therapy. Cancer Res 63: 6815-6824, 2003.

6. Fujiwara T, Shirakawa Y and Kagawa S: Telomerase-specific oncolytic virotherapy for human gastrointestinal cancer. Expert Rev Anticancer Ther 11: 525-532, 2011.

7. Kyo S, Takakura M, Fujiwara T and Inoue M: Understanding and exploiting hTERT promoter regulation for diagnosis and treatment of human cancers. Cancer Sci 99: 1528-1538, 2008.

8. Kong BH, Song Y, Ma DX, Qu X and Jiang S: In vitro treatment of ovarian cancer cells with cytosine deaminase-thymidine kinase fusion disuicide gene therapy system driven by human telomerase reverse transcriptase gene promoter. Zhonghua $\mathrm{Fu}$ Chan Ke Za Zhi 39: 390-395, 2004 (In Chinese).

9. Song Y, Xia Z, Shen K and Zhai X: Autocatalytic caspase-3 driven by human telomerase reverse transcriptase promoter suppresses human ovarian carcinoma growth in vitro and in mice. Int J Gynecol Cancer 23: 642-649, 2013.

10. Kagawa S, Gu J, Swisher SG, Ji L, Roth JA, Lai D, Stephens LC and Fang B: Antitumor effect of adenovirus-mediated Bax gene transfer on p53-sensitive and p53-resistant cancer lines. Cancer Res 60: 1157-1161, 2000.

11. Li JT, Bian K, Zhang AL, Kim DH, Ashley WW, Nath R, McCutcheon I, Fang B and Murad F: Targeting different types of human meningioma and glioma cells using a novel adenoviral vector expressing GFP-TRAIL fusion protein from hTERT promoter. Cancer Cell Int 11: 35, 2011.

12. Hwang do W, Kang JH, Jeong JM, Chung JK, Lee MC, Kim $\mathrm{S}$ and Lee DS: Noninvasive in vivo monitoring of neuronal differentiation using reporter driven by a neuronal promoter. Eur J Nucl Med Mol Imaging 35: 135-145, 2008.

13. Watanabe M, Ueki H, Ochiai K, Huang P, Kobayashi Y, Nasu Y, Sasaki K, Kaku H, Kashiwakura Y and Kumon H: Advanced two-step transcriptional amplification as a novel method for cancer-specific gene expression and imaging. Oncol Rep 26: 769-775, 2011.

14. Chen IY, Gheysens O, Li Z, Rasooly JA, Wang Q, Paulmurugan R, Rosenberg J, Rodriguez-Porcel M, Willmann JK, Wang DS, Contag CH, Robbins RC, Wu JC and Gambhir SS: Noninvasive imaging of hypoxia-inducible factor- $1 \alpha$ gene therapy for myocardial ischemia. Hum Gene Ther Methods 24: 279-288, 2013.

15. Wu L, Johnson M and Sato M: Transcriptionally-targeted gene therapy to detect and treat cancer. Trends Mol Med 9: 421-429, 2003. 
16. Adams JY, Johnson M, Sato M, Berger F, Gambhir SS, Carey M, Iruela-Arispe ML and Wu L: Visualization of advanced human prostate cancer lesions in living mice by a targeted gene transfer vector and optical imaging. Nat Med 8: 891-897, 2002.

17. Alemany R and Curiel DT: CAR-binding ablation does not change biodistribution and toxicity of adenoviral vectors. Gene Ther 8: 1347-1353, 2001.

18. Tao N, Gao GP, Parr M, Johnston J, Baradet T, Wilson JM, Barsoum $J$ and Fawell SE: Sequestration of adenoviral vector by Kuffer cells leads to a nonlinear dose response of transduction in liver. Mol Ther 3: 28-35, 2001.

19. Morral N, O'Neal WK, Rice K, Leland MM, Piedra PA, Aguilar-Córdova E, Carey KD, Beaudet AL and Langston C: Lethal toxicity, severe endothelial injury, and a threshold effect with high doses of an adenoviral vector in baboons. Hum Gene Ther 13: 143-154, 2002.

20. Zhang Y, Chirmule N, Gao GP, Qian R, Croyle M, Joshi B, Tazelaar J and Wilson JM: Acute cytokine response to systemic adenoviral vectors in mice is mediated by dendric cells and macrophages. Mol Ther 3: 697-707, 2001.
21. Yang Y, Su Q and Wilson JM: Role of viral antigens in destructive cellular immune responses to adenovirus vector-transduced cells in mouse lungs. J Virol 70: 7209-7212, 1996.

22. Jooss K, Yang Y, Fisher KJ and Wilson JM: Transduction of dendritic cells by DNA viral vectors directs the immune response to transgene products in muscle fibers. J Virol 72: 4212-4223, 1998.

23. Brand K, Löser P, Arnold W, Bartels T and Strauss M: Tumor cell-specific transgene expression prevents liver toxicity of the adeno-HSV-tk/GCV approach. Gene Ther 5: 1363-1371, 1998.

24. Pastore L, Morral N, Zhou H, Garcia R, Parks RJ, Kochanek S, Graham FL, Lee B and Beaudet AL: Use of a liver-specific promoter reduces immune response to the transgene in adenoviral vectors. Hum Gene Ther 10: 1773-1781, 1999. 